\title{
Application of the method of spatial point pattern analysis to the horizontal spatial distribution of preferential flow paths
}

\author{
by Wenxing $\mathrm{Lu}^{1,2}$, Jinhua Cheng ${ }^{1}$, Wei Wang ${ }^{3}$, Hongjiang Zhang ${ }^{1}$ and Hongwen Zhou ${ }^{2}$
}

\begin{abstract}
Preferential flow significantly influences hydrological processes in forests. The occurrence and development of this flow is directly affected by its spatial distribution. To determine whether or not point pattern analysis method can be used to examine the horizontal spatial distribution of preferential flow paths, experiments were conducted with dye tracer permeation to observe flow processes. Results indicated that an increase in penetration water volume exerted only a specific effect on preferential flow paths of large class in the topmost soil. Moreover, such paths showed distinct clumped patterns at the $25-\mathrm{cm}$ scale under both high permeation water volume and low permeation water volume treatments. Nonetheless, the distribution pattern became uniform as scale range increased. The significance of the correlation between the spatial distribution of preferential flow paths and plant roots decreased from the top soil layer to the bottom. These findings suggest that soil depth and water permeation volume are important to the horizontal spatial distribution of preferential flow paths. Moreover, point pattern analysis method is suitable for investigating the horizontal spatial distribution of preferential flow paths and determining the correlation between the spatial distribution of preferential flow paths and plant roots.
\end{abstract}

Key words: preferential flow paths, brilliant blue dye tracing, spatial point pattern analysis

\begin{abstract}
RESUME
Lécoulement préférentiel influence de façon significative les processus hydrologiques du milieu forestier. L'occurrence et le développement de cet écoulement est directement affecté par sa distribution spatiale. De façon à déterminer si oui ou non l'analyse du motif de points peut être utilisée pour étudier la distribution spatiale horizontale des réseaux d'écoulement préférentiel, des essais de diffusion ont été réalisés au moyen de colorants traceurs afin de suivre les processus découlement. Les résultats ont indiqué qu’une augmentation du niveau de pénétration de l’eau exerçait un effet spécifique seulement sur les réseaux d’écoulement préférentiel de grande envergure situés dans le sol de surface. De plus, ces réseaux ont affichés des schémas agrégatifs distincts à léchelle de $25 \mathrm{~cm}$ à la fois en présence d’un fort volume d’eau de pénétration et d'un faible volume d’eau de pénétration. Néanmoins, le schéma de distribution est devenu uniforme avec l'augmentation de léchelle. L'importance de la corrélation entre la distribution spatiale des réseaux découlement préférentiel et les racines des plantes diminuait à partir de la couche de sol superficiel vers le bas. Ces observations indiquent que la profondeur du sol et le volume d'eau de pénétration sont importants au niveau de la distribution spatiale horizontale des réseaux découlement préférentiel. De plus, l’analyse du motif par points permet létude de la distribution spatiale horizontale des réseaux d'écoulement préférentiel et de déterminer la corrélation entre la distribution spatiale des réseaux découlement préférentiel et les racines des plantes.
\end{abstract}

Mots clés : réseaux découlement préférentiel, traçage par colorant bleu brillant, analyse du motif de points

\section{Introduction}

Preferential flow refers to the uneven and rapid movement of water and solutes through a porous medium, typically soil (Bouma 1981). The channels through which soil water and solutes are conducted preferentially are called preferential flow paths (Helling and Gish 1991). Preferential flow may significantly impact soil water movement in forest soils (Noguchi et al. 1999). This flow can also increase soil water availability and limit surface runoff of forested watersheds. These effects are conducive to the conservation and regulation of water resources. Research on preferential flow and its paths in forest soil assists in improving protection and recov- ery technology systems for forest ecosystems. The image analysis method has been widely used to describe the distribution of preferential flow paths and to extract the corresponding spatial location, the size class of preferential flow paths, and the quantities of independently stained areas in horizontal stained profiles. The horizontal spatial distribution of preferential flow paths refers to the space allocation and distribution state of the different forms of such paths in horizontal dye profiles. The spatial distribution of preferential flow paths in forest soils reflects the heterogeneity of soil structure and also affects the forming process of preferential flow. Thus, the current study can serve as a reference for further investigation

\footnotetext{
${ }^{1}$ College of Soil and Water Conservation, Beijing Forestry University, 35\# Qinghua E. Rd., Haidian district, Beijing, China. Corresponding author's e-mail: jinhua_cheng@126.com.

${ }^{2}$ Yellow River Institute of Hydrology and Water Resources, 12\# Chengbei Rd. E., Jinshui district, Zhengzhou, China.

${ }^{3}$ China Research Center for Environmental Protection and Transportation Safety, China Academy of Transportation Sciences, 240\# Huixinli Rd., Chaoyang district, Beijing, China.
} 
into the spatial heterogeneity and formation mechanism of these paths. Nonetheless, quantitative research has generally been difficult to conduct on the horizontal spatial distribution of preferential flow paths with spatial statistical parameters. The use of a statistical method is complicated as well. The minimum distances among preferential flow paths have been applied as statistical parameters and preliminary analysis was performed by Droogers et al. (1998) on the horizontal spatial distribution of such paths using a frequency statistical method. This method was also employed by Weiler and Flühler (2004). At present, however, research on the horizontal spatial distribution of preferential flow paths is limited because of the difficulty of determining the spatial position message of preferential flow paths in a horizontal soil profile. An accurate spatial analysis method has yet to be established for this research field. If horizontal stained soil profiles at different depths are considered to be an entire space and dispersive preferential flow paths are regarded as individuals within this space, then the spatial point pattern analysis method can be employed in systematic research on the horizontal spa-

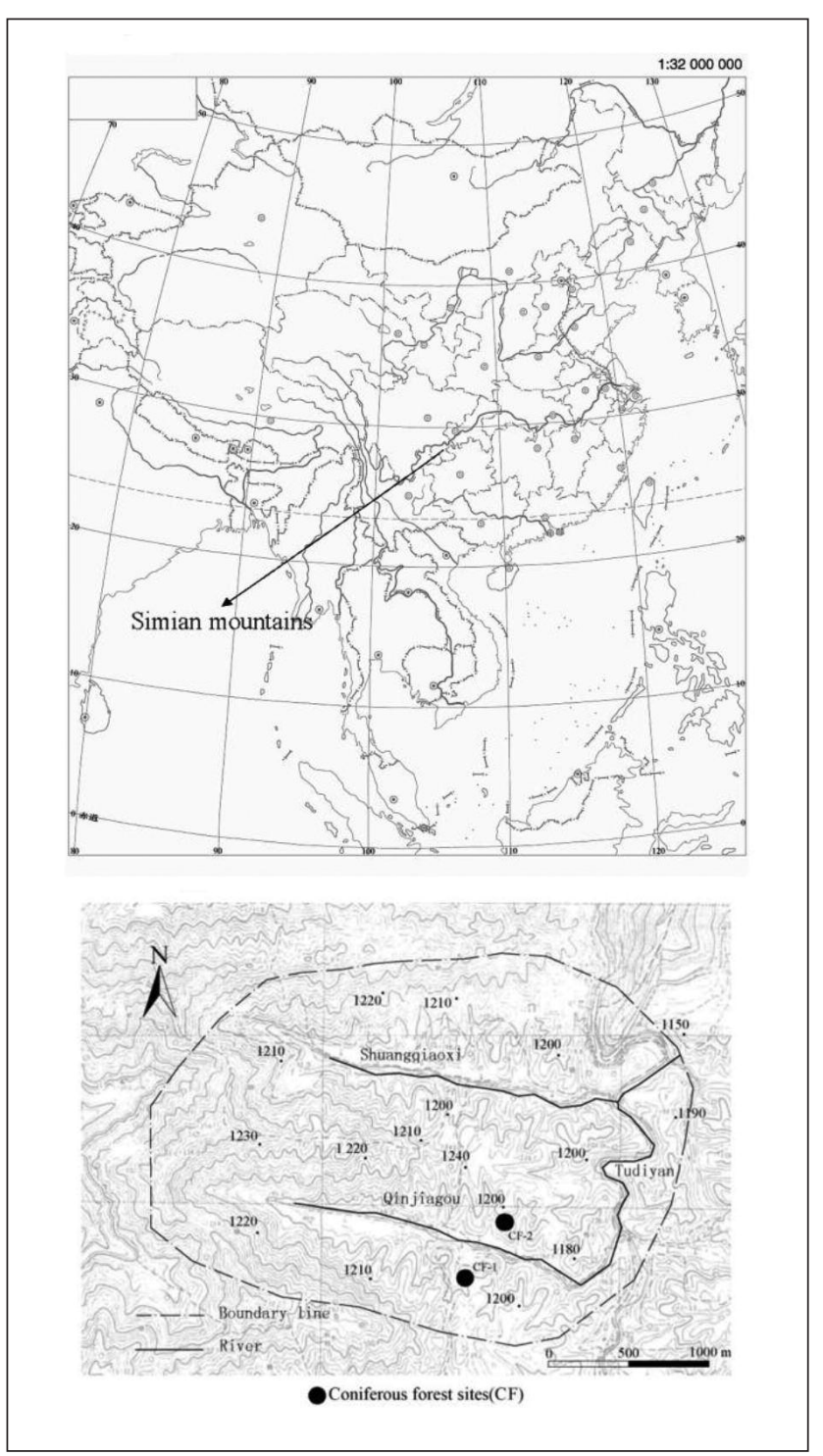

Fig. 1. Location of the experimental sites tial distribution of these paths. Ripley's $K(x)$ function has been utilized in an attempt to quantitatively analyze preferential flow paths of different size classes in forest horizontal soil profiles. In the process, the horizontal spatial distribution state of preferential flow paths can be identified. The spatial distribution state of individuals can be random, clumped, and uniform. A method that can confirm the distribution pattern of individuals is point pattern analysis. This technique can quantitatively show the spatial distribution characteristics of individuals. Nonetheless, the formation process and the mechanism of spatial distribution pattern for individuals in the research space can be examined further (Zhang and Zhao 2008).

Most studies have determined a significant correlation between the formation of preferential flow paths and the growth of plant roots (Mitchell et al. 1995, Gish et al. 1991, 1998; Cheng et al. 2006, 2011). If the formation of preferential flow paths is associated with plant roots, then the spatial distribution of these paths may be correlated to a certain extent as well. Lu (2009) performed multivariate point pattern analysis to identify the influence of plant growth on the formation of preferential flow paths according to the spatial message of preferential flow paths with different size classes.

The objectives of the current study are: (i) to describe the distribution characteristics of preferential flow paths; and, (ii) to determine whether point pattern analysis methods can be used to analyze the horizontal spatial distribution of such paths, and the correlation between the spatial distribution of preferential flow paths and plant roots.

\section{Materials and Methods Experimental setup}

The experiment was conducted in 2010 at two sites in the coniferous forests of Qinjiagou Watershed (Fig. 1). The altitude was $1100 \mathrm{~m}$, the slope $30^{\circ}$ and oriented in the northwest direction. The dominant tree species is Cunninghamia lanceolate (Lamb.) Hook. Both sites are 10-year-old secondary forests. The canopy density is 0.6 , and the degree of cover by shrub and herb layers is $35 \%$. The soils on all sites have the same texture (sandy loam) and initial moisture (Cheng et al. 2011).

\section{Detection of preferential flow paths}

Brilliant blue FCF $\left(4 \mathrm{~g} \times \mathrm{L}^{-1}\right)$ was used as a dye tracer to detect preferential flow paths. The dye experiment was conducted in a $100 \mathrm{~cm} \times 100 \mathrm{~cm}$ flat area. An iron frame with a volume of $70 \mathrm{~cm} \times 70 \mathrm{~cm} \times 50 \mathrm{~cm}$ (length $\times$ width $\times$ height) and a thickness of $0.5 \mathrm{~cm}$ was embedded into the soil at a depth of $30 \mathrm{~cm}$. The soil surface within $5 \mathrm{~cm}$ of the interior and the exterior of the iron wall was compacted to keep the dye tracers within the frame (Cheng et al. 2011). Water was supplied by a Marriott bottle through ponding permeation. After 24 hours, the experimental plot was excavated in five horizontal, $10-\mathrm{cm}$ deep cross sections $(50 \mathrm{~cm} \times 50 \mathrm{~cm})$. The horizontal cross section of each layer was photographed with a digital camera (2560 pixels $\times 1960$ pixels). (Cheng et al. 2011, Cheng et al. 2014). Brilliant blue FCF was applied to the two sites at dosages of $13 \mathrm{~L}$ [equivalent to $25 \mathrm{~mm}$ precipitation $(P=0.05)$ ] and $30 \mathrm{~L}$ [equivalent to $60 \mathrm{~mm}$ precipitation $(P=0.01)$ ]. These treatments were defined as low permeation water volume (LPWV) and high permeation water volume (HPWV), respectively. 


\section{Image analysis}

Image analysis was completed in four steps, as shown in Fig. 2. Three software programs were used, namely: ERDAS IMAGINE 9.2, Adobe Photoshop CS2, and Image-Pro Plus 6.0. The details are described as follows:

\section{(1) Geometric correction}

Given that we cannot easily guarantee that the camera lens and the soil profile are absolutely parallel when gathering images under a restricted shooting environment, the camera may also demonstrate a certain space geometric distortion. Therefore, the original image demonstrates a certain degree of space distortion. ERDAS IMAGINE 9.2 was utilized to correct these geometrical distortions.

The format of the original image was transformed from JPG to IMG via ERDAS software, and the polynomial operation method was selected in the Image Geometric Correction function. Based on the scale size of the original image, any three sidelines and corners of the dyed section area were used as the control points and the plane coordinates were defined based on the image length. The position of the fourth control point was identified automatically by the software. The cubic convolution method was adopted to resample the image. The geometric-corrected image was exported with an RGB pattern and in JPG format. Following the requirements of the specific analysis accuracy, the resolution of the output image was 5 pixel/cm-1 (Weiler and Flühler 2004). Therefore, the size of the horizontal section dyeing image after geometric correction was $1000 \times 1000$ pixels.

\section{(2) Illumination correction}

The difference in the sensitivity of the dyeing profile surface will result in uneven lighting and shading of the original image. If the parsing and analysis of the horizontal section dyeing image was directly performed after the geometric correction with RGB pattern, normal soil under poor light could be misinterpreted as stained soil. Therefore, illumination correction must be performed.

A standard grayscale colorimetric card was placed next to the dyeing profile when taking photos. The light sensitivity of the grayscale ribbons consistently ranged between 0 and 255. The average color, brightness, contrast, and exposure levels from the standard grayscale colorimetric card were used as reference. The color, brightness, contrast, and exposure levels in the Image Adjust option of Adobe Photoshop CS3 were adopted for the illumination correction.

\section{(3) Color adjustment}

The RGB image contains three colors of red, green, and blue. To simplify the image information and to identify the shape of the dyeing area, the image is converted into a bitmap image via color adjustment method. Adobe Photoshop CS3 was used for the sampling analysis of the stained soil in the image to confirm the dyeing area of the color range. The Color Replace function in the image adjust option was used to replace the stained soil areas to white $(P R=P G=P B=255)$. Afterward, the RGB image was converted to grayscale, and the Reverse function was adopted to transform the grayscale image into a colored

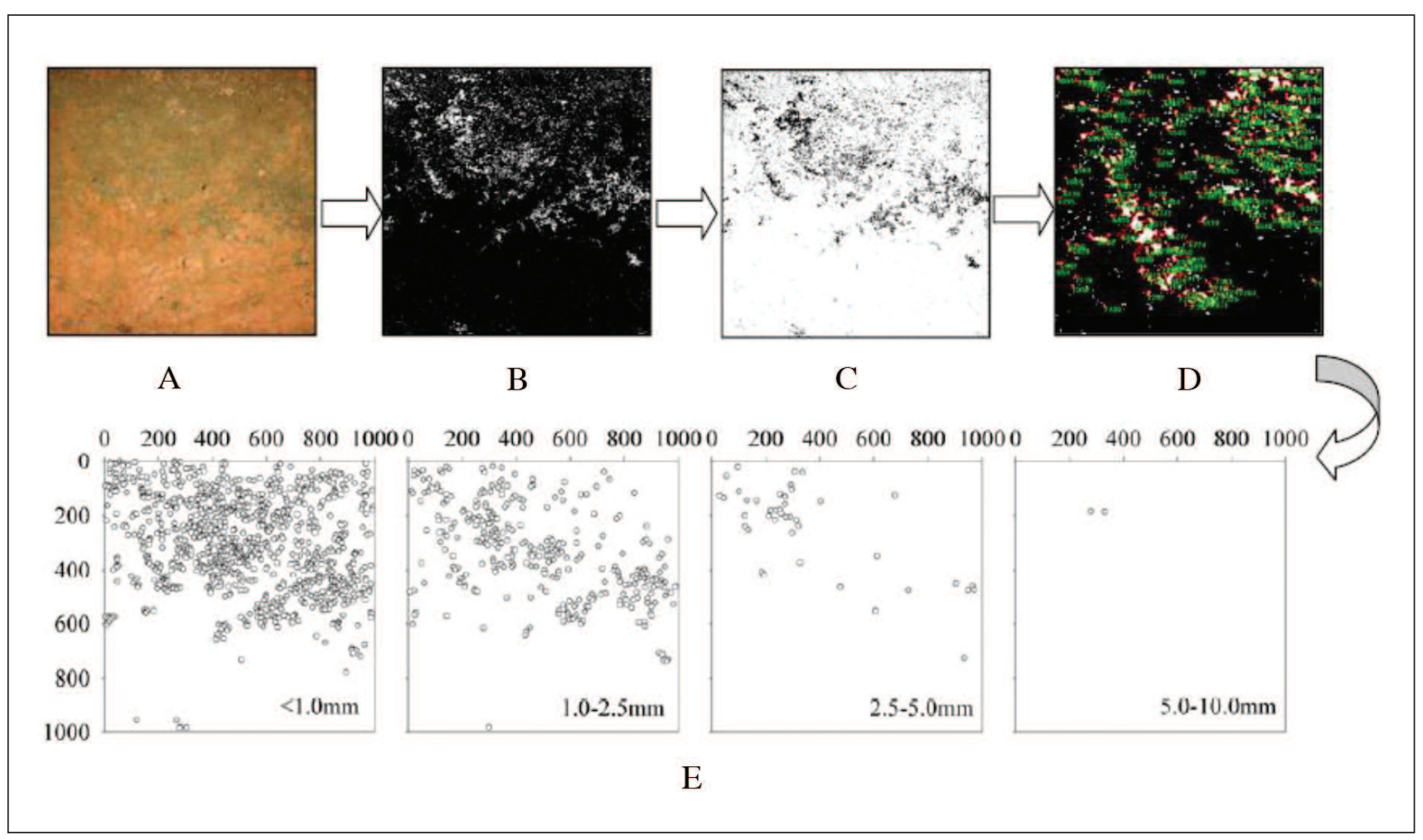

Fig. 2. Visual representation of the image processing steps used to create the horizontal profile (50 cm wide by $50 \mathrm{~cm}$ long) images for further analysis. A: original image; B: the image after correction for lighting and geometrical distortion; C: a binary image after applying the dye detection algorithm; $\mathbf{D}$ : the image after reducing noise from the processes of color adjustment and format conversion, and the dyed area was calculated accurately; and, E: location and quantities of preferential flow paths of different size classes. 
image. The stained areas were marked in black, whereas unstained areas were marked in white or grey-white.

The Threshold function was used to adjust the magnitude of the threshold and the color levels, which would make the dyeing area of the image after the color correction process consistent with the original image. After treating with the grayscale pattern, the image was converted into a bitmap image with TIFF format via the diffusion and color imitation method.

\section{(4) Noise reduction}

Noise appeared on the image during the color adjustment and format conversion processes, thereby affecting the image resolution precision. Therefore, the Image Filter function, which was based on mathematical morphology, should be adopted for noise reduction. The Morphological Opening Filter function in Image-Pro Plus 6.0 was also adopted. Corrosion operation was performed to remove the independent noise, and then a dilation operation performed to recover the boundary of the dyeing image area. Given the limitations of the software function, the stained soil in the bitmap image after noise reduction was marked in white, whereas the unstained soil was marked in black. Noise reduction can effectively reduce the computational complexity of the characteristic parameter extraction of the image and simplify the image parsing process.

\section{(5) Numerical value transform of image}

The processed bitmap image was actually a numerical matrix containing the values 0 and 255. Numerical value transformation should be performed to extract the image feature parameters. The Bitmap Analysis function of the Image-Pro Plus 6.0 software was adopted to convert the image after noise reduction into a bitmap numerical matrix $(1000 \times 1000)$, which would then be exported into Excel format. The matrix contained only two numerical values, of which 255 (white) denoted the stained soil and 0 (black) the unstained soil.

\section{(6) Image characteristic parameter parsing}

The Classification and Count function in Image-Pro Plus 6.0 was used to distinguish size classes from the spatial position message of the preferential flow paths in the stained area. The "watershed" method (Vincent and Soille 1991) was then performed to allocate the connective stained area to independent individuals. The closed group and the acreage and areal coordinates of the dyeing area were counted. The equivalent radius of influence zone of the independent preferential paths (radius of influence) can be calculated through the back calculation of the circular area formula. The recorded quantities of these paths in four size classes were $\leq 1.0 \mathrm{~mm}, 1.0-2.5 \mathrm{~mm}$, $2.5-5.0 \mathrm{~mm}$, and 5-10 $\mathrm{mm}$. The artificial statistics method was used to determine the position of the roots by observing root coordinates in the horizontal image with the naked eye following illumination correction.

\section{Spatial point pattern analysis}

Spatial point pattern analysis considers all point-to-point (preferential flow paths to preferential flow paths) distances in describing two-dimensional distribution patterns. The spa- tial patterns were analyzed using Ripley's $L(x)$ function, which is a transformation of Ripley's K-function (Diggle 1983, Ripley 1981). In spatial point pattern analysis, a circle with radiusis $x$ located at the centre of each point. Furthermore, the other points within the circle are counted. The method can be employed to measure the distances of all inter-preferential flow paths, as well as the spatial distribution patterns at various distance scales $L(x)$ is obtained by calculating relative density $[K(x)]$ of all plants within the circle (Ripley 1981):

(1) $K(x)=\left(\frac{A}{n^{2}}\right) \sum_{i=1}^{n} \sum_{j=1}^{n} \frac{1}{W_{i j}} I_{x}\left(u_{i j}\right)$

$(\mathrm{i} \neq \mathrm{j})$,

Where $A$ is the area of the plot; $n$ the number of preferential flow paths for a radius; $x$ the distance (radius) scale; and $\sum_{i=1}^{n} \sum_{j=1}^{n} \frac{1}{W_{i j}} I_{x}\left(u_{i j}\right)$ represents the conspecific numbers within a distance shorter than $x, I_{x}$ is the summation of the number of points and $W$ the weighting factor for the edge corrections. Given that $K(x)=\pi x^{2}$ when the preferential flow paths are randomly arranged in a Poisson-distributed soil profile, the distribution state of the individuals are shown by the $L(x)$ function which is the transformation of Ripley's $K(x)$ function:

$$
\text { (2) } L(x)=\sqrt{K(x) / \pi}-x
$$

Monte Carlo simulation was used to test the statistical significance of the deviations of $L(x)$ from zero under the null hypothesis of a complete random distribution (Besag and Diggle 1977). Confidence intervals of $99 \%$ were generated using high and low values of $L(x)$ derived from 2000 simulations of random permutations. The maximum and minimum values of $L(x)$ vary at each distance and across all simulations; these values form the upper and lower confidence bounds known as the simulation envelopes. The spatial pattern was observed across a range of $25-\mathrm{cm}$ spatial scales (half the side length of the stained horizontal profile). If the $L(x)$ of the studied individuals in these spatial scales lies between the two confidence bounds, then the distribution is random. By contrast, the distribution is either clumped or uniform if the value is either higher than the upper confidence bound or lower than the lower confidence bound. Spatial point pattern method employs Ripley's $K(x)$ function through ADE-4 software (Goreaud and Pélissier 1999).

\section{Multivariate point pattern analysis}

The analysis of the correlation between plant roots and the horizontal spatial distribution of preferential flow paths was based on the Ripley'sfunction in combination with multivariate point pattern analysis to explore the distribution relationship of the two individuals up to distance $x$ (Diggle 1983). If the roots and the horizontal spatial distribution of preferential flow paths are mutually independent of each other, then the corresponding bivariate equation is as follows:

(3) $K_{12}(x)=\left(\frac{A}{n_{1} n_{2}}\right) \sum_{i=1}^{n_{1}} \sum_{j=1}^{n_{2}} \frac{1}{W_{i j}} I_{x}\left(u_{i j}\right)$

$(i \neq j)$, 
Where $n_{1}$ and $n_{2}$ represent the number of plant roots and preferential flow paths, respectively; $A, I_{x}\left(u_{i j}\right)$, and $W_{i j}$ are defined as in Formula (1); and, $i$ and $j$ correspond to plant roots and preferential flow paths of the individuals in a certain soil profile, respectively. Equation (3) may be transformed into:

(4) $L_{12}(x)=\sqrt{K_{12}(x) / \pi}-x$

As expected, $L_{12}(x)>0$ indicates that, on average, pattern 2 has more points than pattern 1 within distance in the bivariate case as a result of independence. This outcome indicates the attraction (competition) between the two patterns up to distance $x$. Similarly, the values of $L_{12}(x)<0$ imply the repulsion (facilitation) between the two patterns up to distance $x$. $L_{12}(x)=0$ indicates independent patterns. To investigate the interactions between plant roots and preferential flow paths, we conducted bivariate analysis under the toroidal shift null model (independence) on two functions. The significant deviation from an underlying null model was also tested through Monte Carlo simulation. If the $L_{12}(x)$ of the studied individuals lies between the two confidence bounds up to distance, then the individuals are regarded as independent of each other. By contrast, if the $L_{12}(x)$ values of these subjects are higher and lower than the upper and lower confidence bounds, then these individuals are positively and negatively correlated, respectively (Wiegand and Moloney 2004, Chao et al. 2008).

\section{Results and Discussion \\ Quantities of preferential flow paths in horizontal profiles}

The preferential flow paths in forest soils were affected by changes in the surrounding environment. The horizontal spatial distribution of these paths varied in different soil profiles (Droogers et al. 1998). On the basis of the detection results, the preferential flow paths of the horizontal stained image could be divided into four size classes: $\leq 1.0 \mathrm{~mm}, 1.0-2.5 \mathrm{~mm}$, $2.5-5.0 \mathrm{~mm}$, and $5.0-10.0 \mathrm{~mm}$. The quantities of the preferential flow paths in different size classes are shown in Table 1. The characteristics of these paths were similar. The summation of the preferential flow paths in the $10-\mathrm{cm}$ and $20-\mathrm{cm}$ soil layers was significantly higher than that in the bottom soil layer. This result indicated that the number of openings increased and that the connected pore structure was distributed in the topmost layer of soil. This layer can conduct soil moisture effectively. The decrease in preferential flow paths in the bottom soil layer also suggested that connectivity decreased with the increase in soil depth. The soil moisture in the $30-\mathrm{cm}, 40-\mathrm{cm}$, and $50-\mathrm{cm}$ soil layers was mainly transported along the preferential flow paths with high connectivity.

The elevated water supply merely increased the quantities of large preferential flow paths on the surface soil layer, especially those of paths with semi-diameters in the ranges of $2.5-5.0 \mathrm{~mm}$ and $5.0-10.0$ $\mathrm{mm}$. The $10-\mathrm{cm}$ soil layer under HPWV treatment contained 2456 and 280 paths with these features; this count exceeded that in the soil under LPWV treatment. This finding indicates that the preferential flow paths are closely related to soil moisture connectivity (Haws et al. 2004).

In summary, the quantities of preferential flow paths exhibited similar tendencies. These quantities decreased with an increase in soil depth, particularly in the $20-\mathrm{cm}$ and $30-\mathrm{cm}$ soil layers. Therefore, the variability in the conductivity of the preferential flow paths in the area was superior to that in the conductivities of other soil layers, and interflow differentiation was more severe in the former. The elevated water supply increased considerably the quantities of preferential flow paths of large size classes in the surface soil, as well as improved the conductivity of these paths.

Horizontal spatial distribution patterns of preferential flow paths The horizontal spatial distribution of preferential flow paths refers to the space allocation and distribution of the different forms of such paths in horizon dye profiles. These profiles reflect the correlations among preferential flow paths. Therefore, this research may serve as a reference for further investigation into the spatial heterogeneity and formation mechanisms of preferential flow paths. Horizontal spatial distribution patterns were also studied in relation to preferential flow paths of four size classes, namely, $\leq 1.0 \mathrm{~mm}, 1.0-2.5$ $\mathrm{mm}, 2.5-5.0 \mathrm{~mm}$, and $5.0-10.0 \mathrm{~mm}$ under HPWV and LPWV treatments at different soil depths.

\section{Horizontal spatial distribution patterns of preferential flow paths under LPWV treatment}

The horizontal spatial distribution patterns of preferential flow paths under LPWV treatment are shown in Fig. 3. The distribution of these paths was clumped at the $25-\mathrm{cm}$ scale, given different size classes of horizontal stained profiles.

At the same scale, flow paths with a semi-diameter of $\leq 1.0$ $\mathrm{mm}$ exhibited distinct clumped patterns in the $10-\mathrm{cm}, 20-\mathrm{cm}$, $40-\mathrm{cm}$, and $50-\mathrm{cm}$ soil layers. At the ${ }^{3} 15-\mathrm{cm}$ scale, distribution was either random or uniform in the $10-\mathrm{cm}$ and $20-\mathrm{cm}$ soil layers under LPWV treatment. At the ${ }^{3} 20-\mathrm{cm}$ scale, the $30-\mathrm{cm}$ soil layer exhibited a random distribution; the transi-

Table 1. Quantities of preferential flow paths of different size classes with different treatment

Quantities of preferential flow paths of different size classes, number per square metre

\begin{tabular}{|c|c|c|c|c|c|c|}
\hline \multirow[b]{2}{*}{ Treatment } & \multirow{2}{*}{$\begin{array}{l}\text { Soil layers } \\
\quad(\mathrm{cm})\end{array}$} & \\
\hline & & Summation & $\leq 1.0 \mathrm{~mm}$ & $1.0-2.5 \mathrm{~mm}$ & $2.5-5.0 \mathrm{~mm}$ & $5.0-10.0 \mathrm{~mm}$ \\
\hline \multirow[t]{5}{*}{ LPWV } & 10 & 26072 & 21100 & 4812 & 156 & 4 \\
\hline & 20 & 17208 & 11616 & 4648 & 892 & 52 \\
\hline & 30 & 11136 & 7140 & 3116 & 804 & 76 \\
\hline & 40 & 8468 & 6392 & 1856 & 208 & 12 \\
\hline & 50 & 2660 & 2292 & 344 & 24 & - \\
\hline \multirow[t]{5}{*}{ HPWV } & 10 & 18000 & 9032 & 6232 & 2456 & 280 \\
\hline & 20 & 16620 & 13012 & 3168 & 400 & 40 \\
\hline & 30 & 7408 & 5876 & 1356 & 172 & 4 \\
\hline & 40 & 1636 & 1284 & 324 & 28 & - \\
\hline & 50 & 3544 & 3024 & 476 & 44 & - \\
\hline
\end{tabular}


$\leqslant 1.0 \mathrm{~mm}$
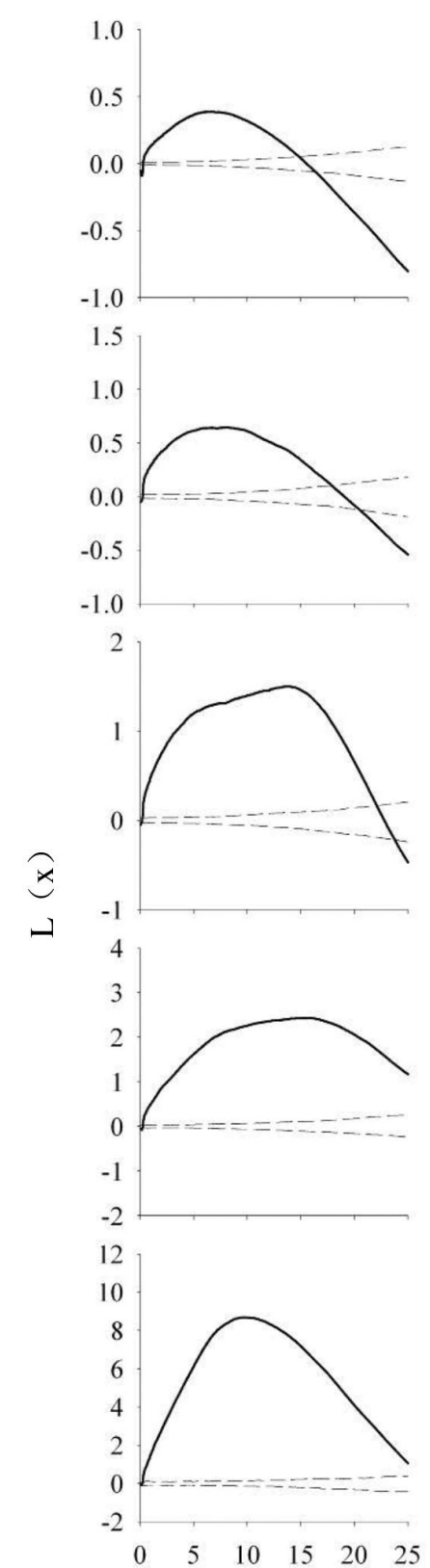

$1.0-2.5 \mathrm{~mm}$
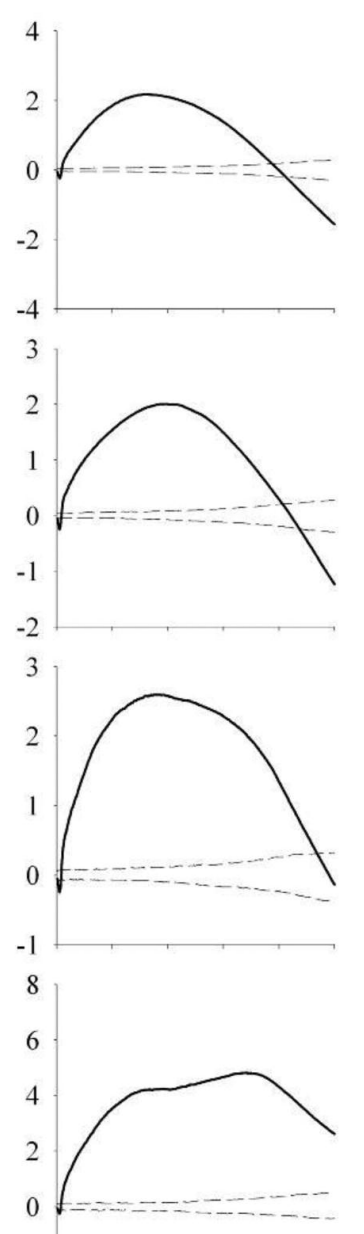

$-2$

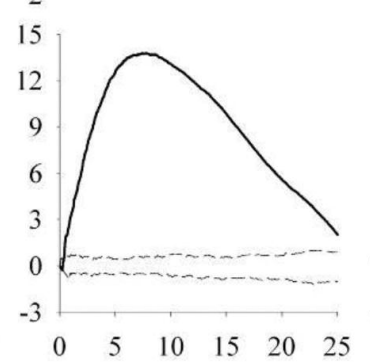

$\mathrm{x}(\mathrm{cm})$
$2.5-5.0 \mathrm{~mm}$

$5.0-10.0 \mathrm{~mm}$
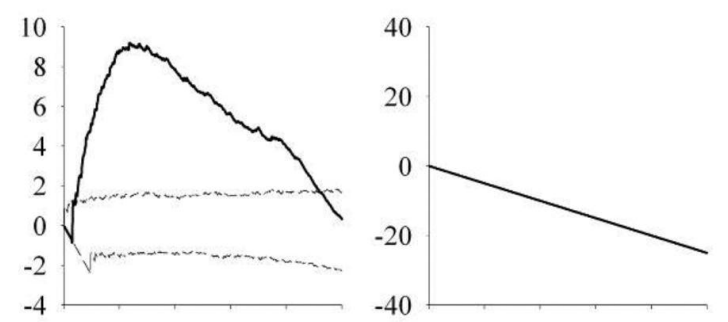

$10 \mathrm{~cm}$
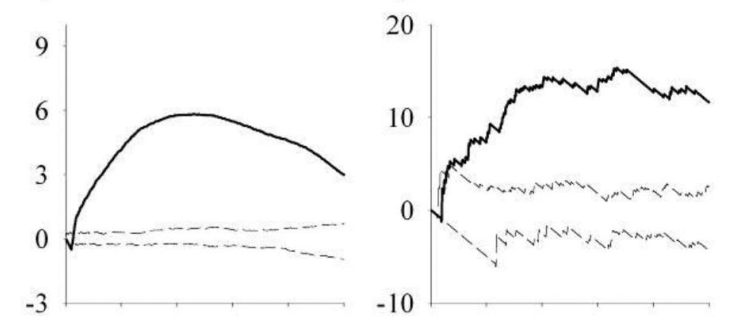

$20 \mathrm{~cm}$
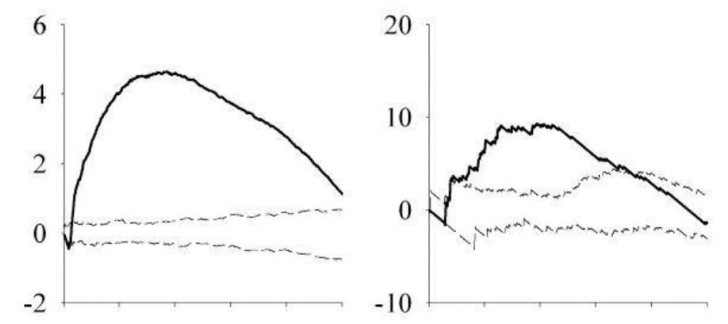

$30 \mathrm{~cm}$
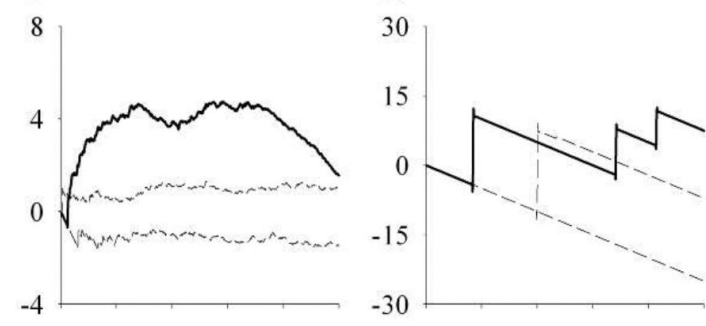

$40 \mathrm{~cm}$

10

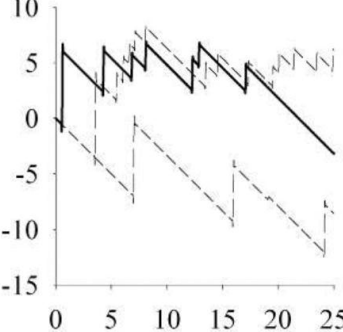
confidence bounds

$\mathrm{L}(\mathrm{x})$

$50 \mathrm{~cm}$

Fig. 3. Spatial distribution patterns of preferential flow paths with LPWV treatment. The sample statistic $L(x)$ is shown as a solid line, the Monte-Carlo simulations as dotted lines.

tion region of preferential flow was located at this layer. Distribution patterns also indicated that both the morphological differentiation of interflow and the spatial heterogeneity of preferential flow were random to a certain degree.

The distribution patterns of preferential flow paths with semi-diameters in the range of $1.0-2.5 \mathrm{~mm}$ were almost identical to those of flow paths with a semi-diameter of $\leq 1.0 \mathrm{~mm}$.
This finding suggests that flow paths with small size classes displayed similar distribution tendencies. The clumping degree of paths with semi-diameters in the range of 1.0-2.5 $\mathrm{mm}$ was slightly lower than that of paths that were $\leq 1.0 \mathrm{~mm}$ in semi-diameter. This finding may be attributed to the increased interaction among preferential flow paths.

Paths with semi-diameters in the range of $2.5-5.0 \mathrm{~mm}$ 

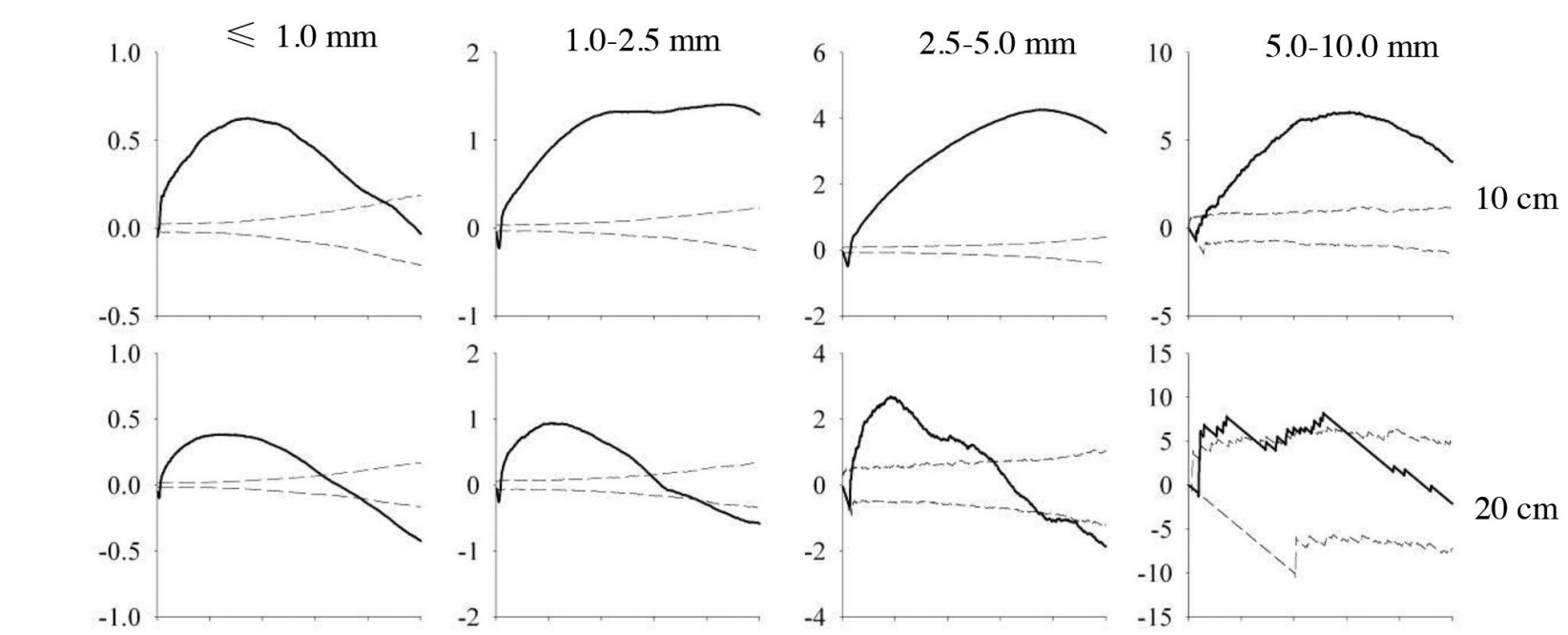

$20 \mathrm{~cm}$
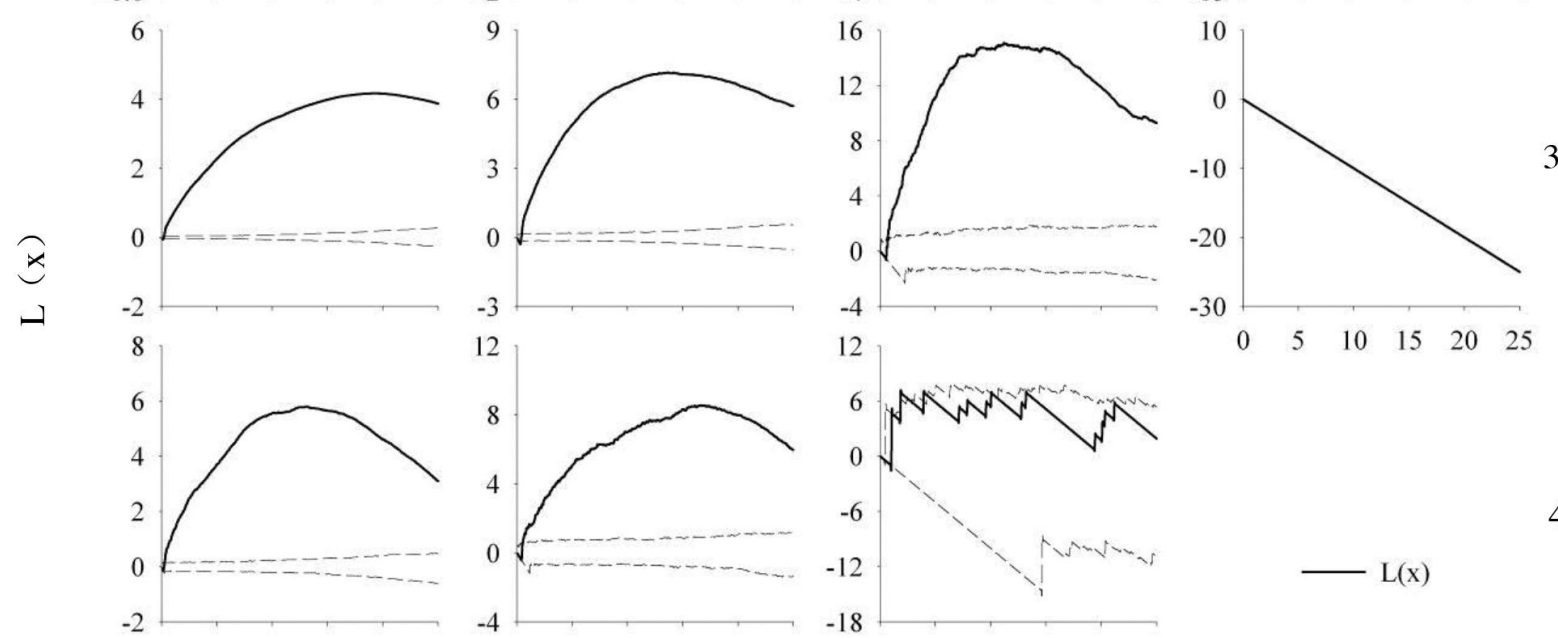

$30 \mathrm{~cm}$
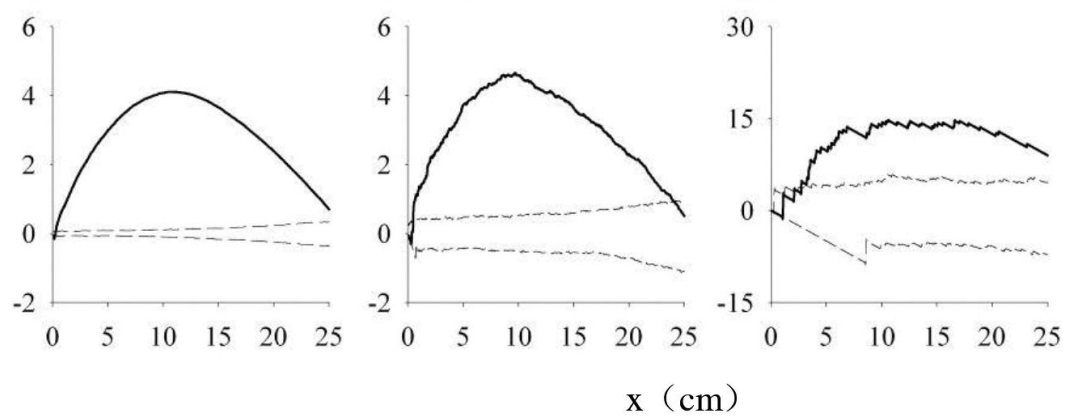

$40 \mathrm{~cm}$

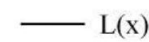

$50 \mathrm{~cm}$

Fig. 4. Spatial distribution patterns of preferential flow paths with HPWV treatment

exhibited a distinct clumped distribution. The distribution pattern of preferential flow paths with a semi-diameter of $\leq 2.5 \mathrm{~mm}$ was identical to that of paths with semi-diameters from $2.5 \mathrm{~mm}$ to $5.0 \mathrm{~mm}$ in the $10-\mathrm{cm}$ and $20-\mathrm{cm}$ soil layers. A random distribution was observed in the $50-\mathrm{cm}$ soil layer; this may be attributed to the enlargement of the pore structure through the incompact fragmental products of bed rock. Few preferential flow paths had semi-diameters in the range of 5.0-10.0 mm; hence, the accuracy of spatial analysis was reduced to some extent. This type of path exhibited clumped distribution in the topmost $10-\mathrm{cm}$ and $20-\mathrm{cm}$ soil layers. Below the $20-\mathrm{cm}$ soil layer, however, distribution was random at the ${ }^{3} 15 \mathrm{~cm}$ scale. Thus, the continuity of preferential flow paths in the vertical direction exhibited spatial heterogeneity. This finding may be related to changes in soil structure and properties. 

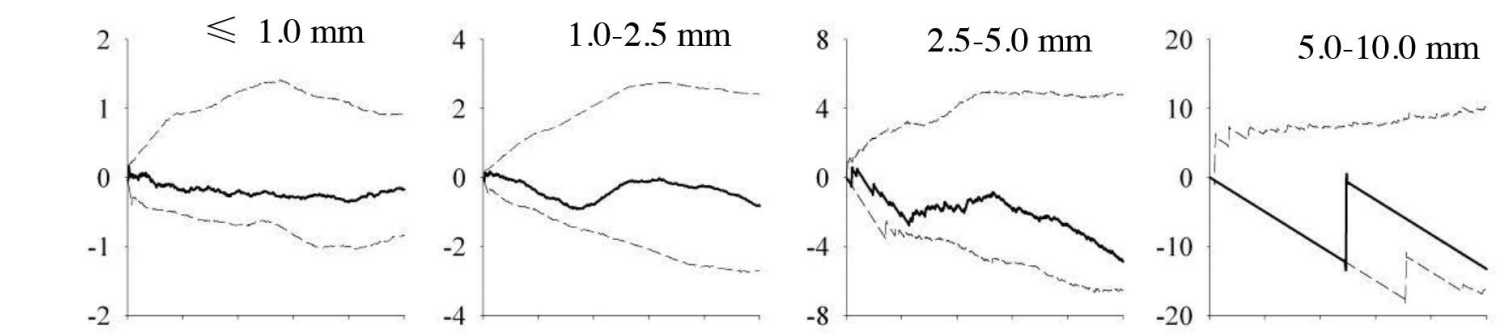

$10 \mathrm{~cm}$
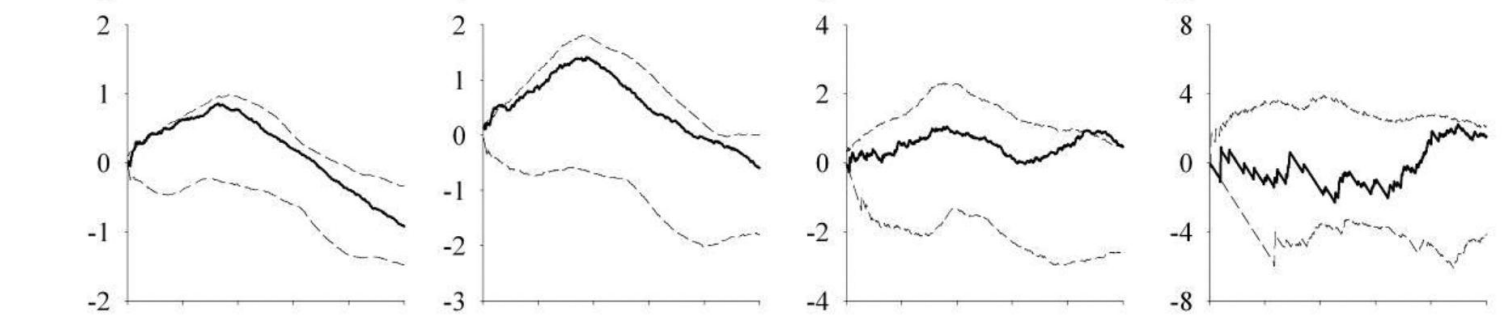

$20 \mathrm{~cm}$
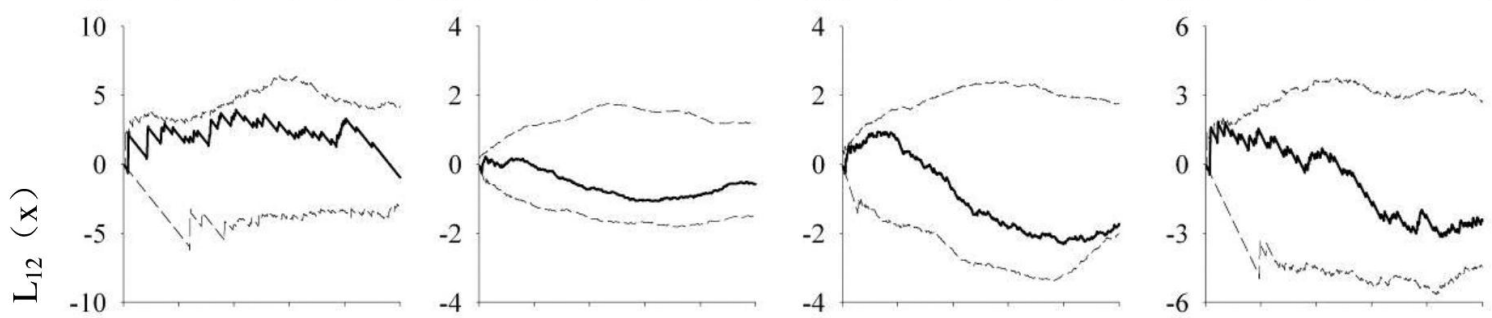

$30 \mathrm{~cm}$
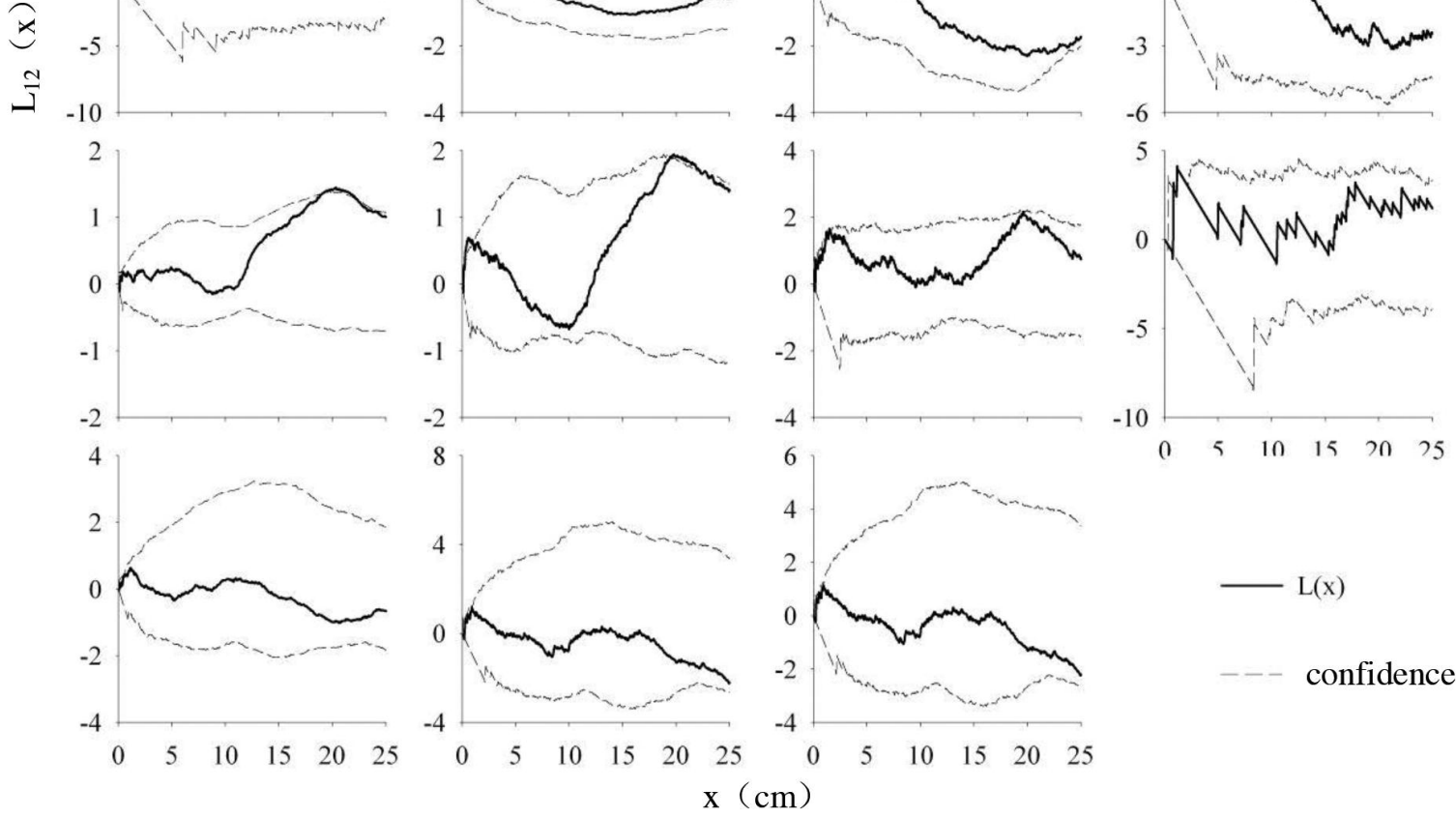

$40 \mathrm{~cm}$

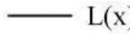

$50 \mathrm{~cm}$

confidence bounds

Fig. 5. Spatial association of preferential flow paths and roots with LPWV treatment

Horizontal spatial distribution patterns of preferential flow paths under HPWV treatment

The preferential flow process in the forest soil profile under HPWV treatment was more intense than that under LPWV treatment because the water supply was increased and the duration of the simulated rainfall was lengthened. The spatial distribution patterns of preferential flow paths under HPWV treatment are depicted in Fig. 4.

All of the horizontal spatial distribution patterns of paths in different soil layers varied under LPWV and HPWV treat- ments. Overall, changes in the horizontal spatial distribution of preferential flow paths were complicated at the $25-\mathrm{cm}$ scale. Paths with a semi-diameter of $\leq 1.0 \mathrm{~mm}$ under HPWV treatment distributed randomly in the $10-\mathrm{cm}$ and $20-\mathrm{cm}$ soil layers at the ${ }^{3} 20$-cm scale.

Preferential flow paths with semi-diameters in the range of $1.0-2.5 \mathrm{~mm}$ were similar to those with a semi-diameter of $\leq$ $1.0 \mathrm{~mm}$.

Paths with semi-diameters in the range of $2.5-5.0 \mathrm{~mm}$ mainly exhibited a clumped distribution. This type of prefer- 

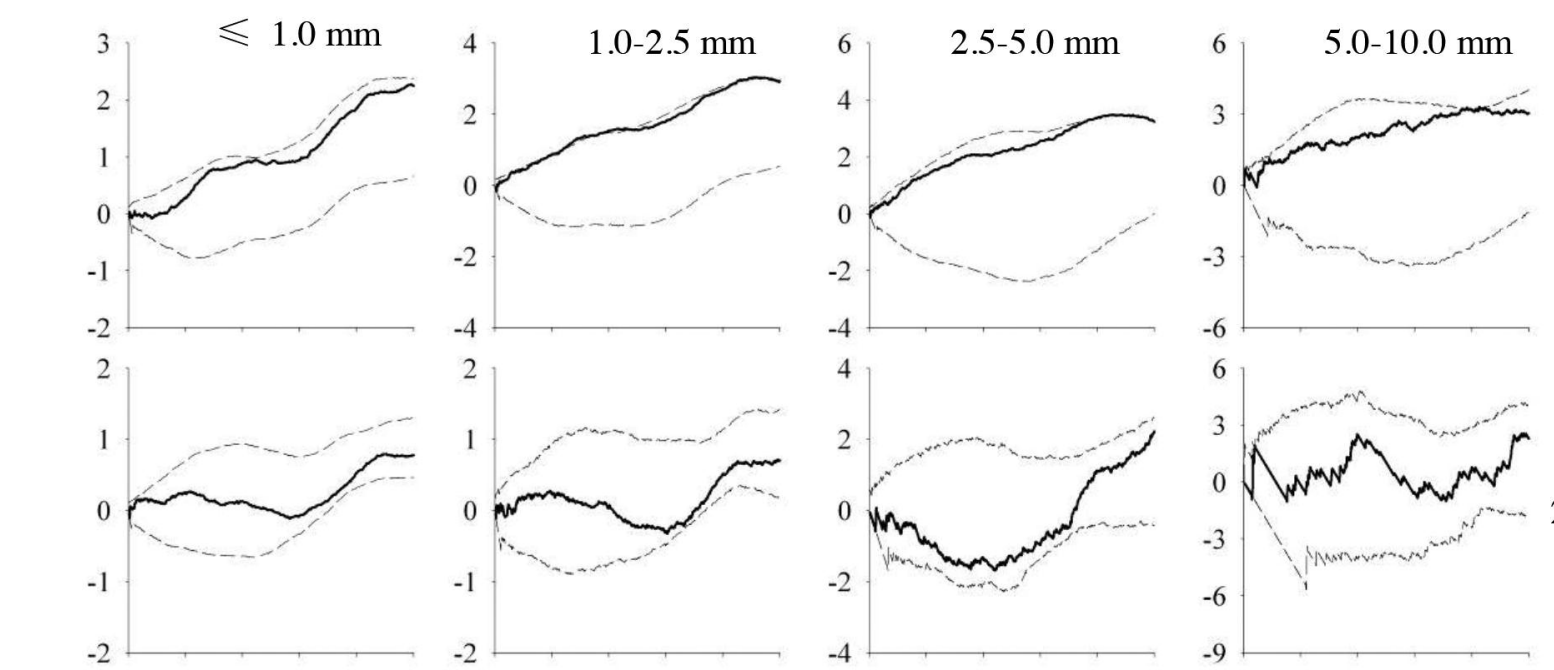

$10 \mathrm{~cm}$
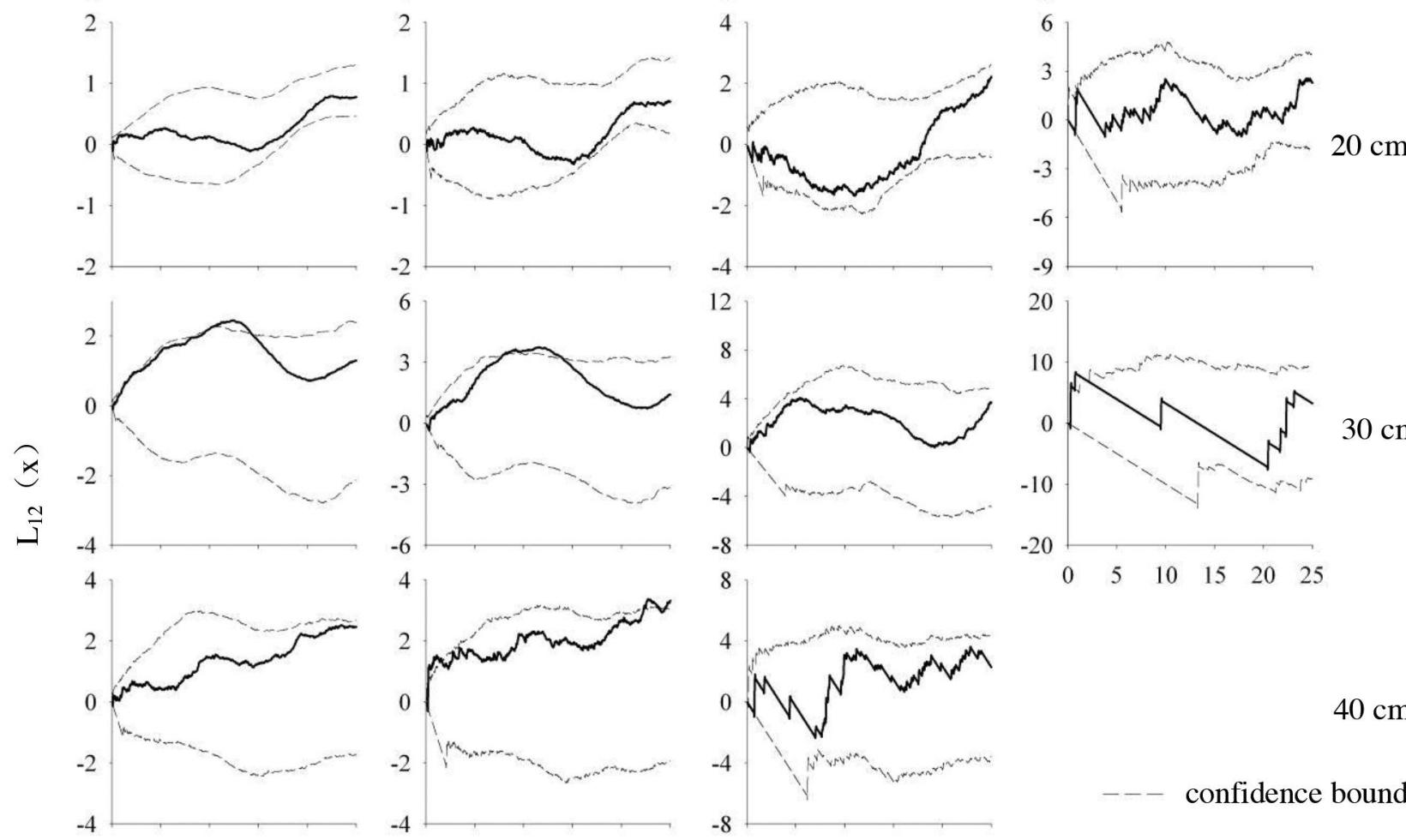

8
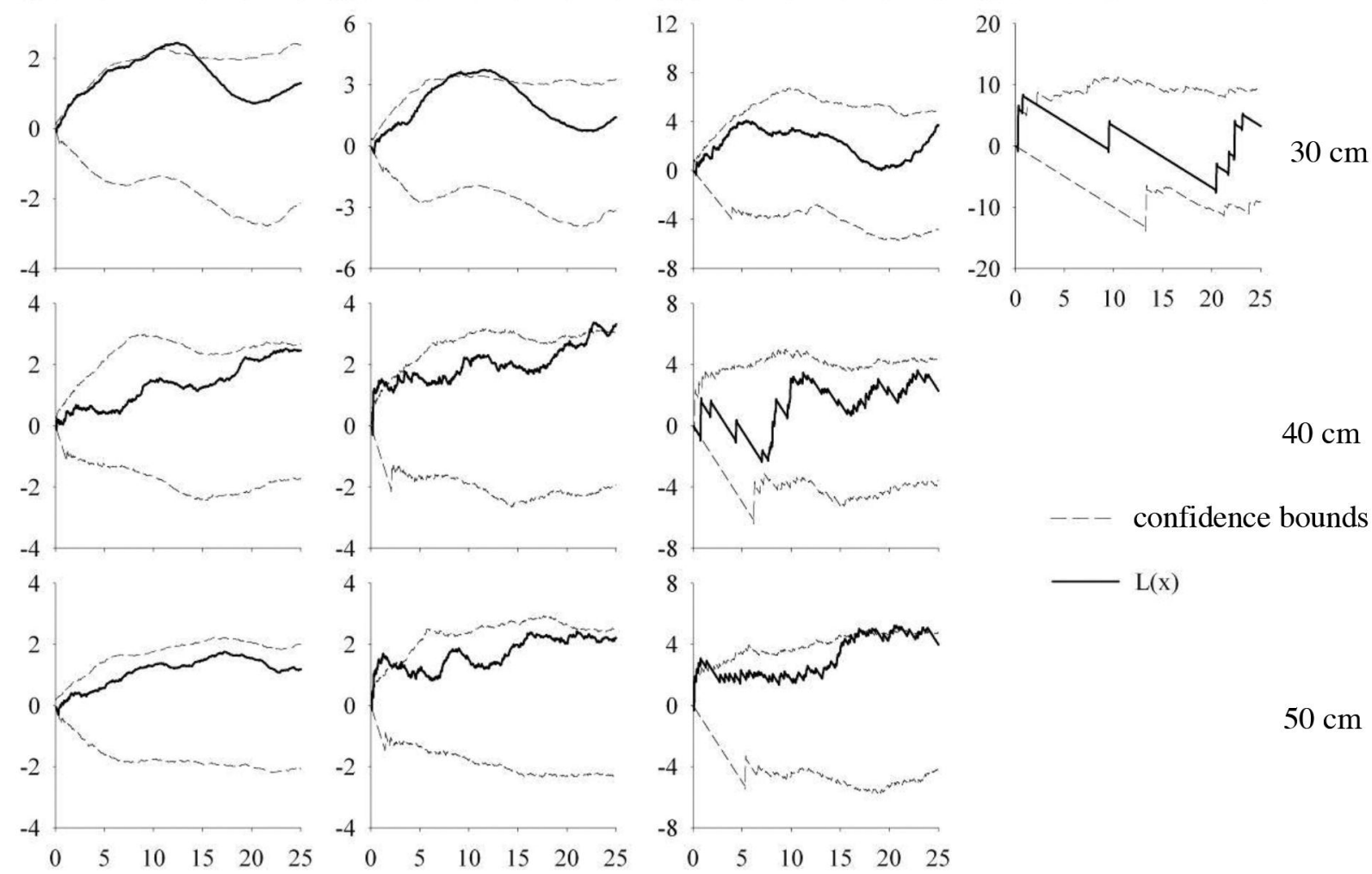

8

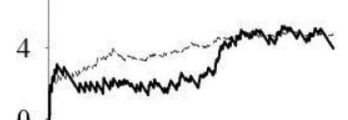

$40 \mathrm{~cm}$
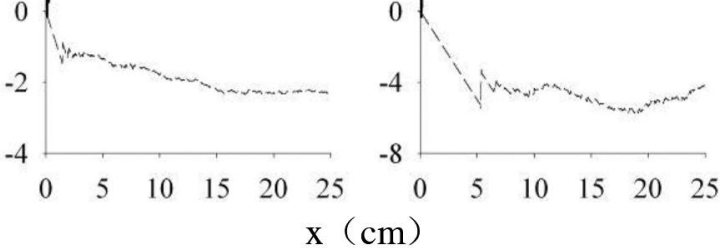

$50 \mathrm{~cm}$

Fig. 6. Spatial association of preferential flow paths and roots with HPWV treatment

ential flow path displayed a random distribution in the bottom layer of soil at the $25-\mathrm{cm}$ scale; however, this tendency weakened progressively.

Although the quantities of preferential flow paths with semi-diameters in the range of $5.0-10.0 \mathrm{~mm}$ were higher under HPWV treatment than under LPWV treatment, the former remains smaller than that obtained by others. Therefore, the spatial analysis results were inaccurate. This type of preferential flow path displayed a clumped distribution under HPWV treatment. The amount of random distribution pat- terns decreased under LPWV treatment. This result indicates that the preferential flow process was intensified further and that preferential flow paths of a large size class mainly exhibited clumped distribution in soil layers when water supply increased.

Regardless of whether HPWV or LPWV treatment was used, the horizontal spatial distribution trend of preferential flow paths was uniform in the horizontal stained profiles when distance scale increased. This result demonstrates that the spatial heterogeneity of forest soil structure is primarily 
related to distribution position and the configuration of plants. If the stand structure is homogeneous and the configuration of plants is steady, then the onset and distribution of preferential flow paths is uniform. This uniformity sustains the property of soil water movement in forest soil as a whole.

\section{Spatial association of plant roots with preferential flow paths}

Artificial record method was employed to obtain the message of plant root position by observing the root coordinates in the image with the naked eye following illumination correction. Moreover, the spatial association of plant roots with preferential flow paths of different size classes $(\leq 1.0 \mathrm{~mm}, 1.0-2.5 \mathrm{~mm}$, 2.5-5.0 $\mathrm{mm}$, and $5.0-10.0 \mathrm{~mm}$ ) was examined through multivariate point pattern analysis. Monte Carlo method was used to calculate the upper and lower confidence bounds $(P=0.01)$ known as the simulation envelopes to verify the spatial distribution relationship between plant roots and preferential flow paths. The spatial association of paths of different size classes and plant roots with HPWV and LPWV treatments is shown in Fig. 5 and Fig. 6.

The spatial association of preferential flow paths with plant roots exhibited significant positive correlations in the $10-\mathrm{cm}, 20-\mathrm{cm}, 30-\mathrm{cm}$, and $40-\mathrm{cm}$ soil layers, especially under HPWV treatment. This result is attributed to the strengthened preferential flow process in the topmost soil layers of the coniferous plots. Furthermore, soil water was transported along the preferential flow paths established by superficial plant roots.

The spatial association of preferential flow paths and plant roots displayed an insignificant correlation in the $50-\mathrm{cm}$ soil layer because the bed rock in this area is severely weathered and fractured. This condition affected the preferential flow process. Moreover, superficial plant roots were difficult to extend to this area.

The spatial association between preferential flow paths of different size classes and plant roots changed substantially at various spatial scales, especially for paths with semi-diameters in the ranges of $1.0-2.5 \mathrm{~mm}$ and $2.5-5.0 \mathrm{~mm}$. This observation may be related to the complex change in the quantities of preferential flow paths in coniferous forests. Nonetheless, the spatial association between plant roots and paths with semi-diameters in the ranges of $1.0-2.5$ and $2.5-5.0 \mathrm{~mm}$ was almost consistent throughout the entire soil profile scale $(x=25 \mathrm{~cm})$.

\section{Reliability of the method for studying distribution patterns of preferential flow paths}

If the soil horizontal dyeing profile of different depths was seen as a spatial integral and the preferential flow paths that were dispersed in the horizontal dyeing profile were seen as individuals of the spatial integral, then the spatial point pattern analysis method in landscape ecology could be used to examine the spatial distribution rule of the preferential flow paths.

This study of the horizontal spatial distribution patterns and the spatial association of preferential flow paths with roots in horizontal stained profiles of different soil layers under HPWV and LPWV treatments showed that the distribution patterns were affected by the profile scale, penetration water volume, and plant roots. The change rule of the distribution patterns of the flow paths conforms to both theory and practice. The preferential flow paths of different size classes showed similar horizontal space distribution patterns. The consistency in the patterns is consistent with the spatial homogeneity hypothesis of Ripley's K-function, which also confirms the reliability of the spatial point pattern analysis in studying preferential flow paths. Therefore, this method can be used to determine the relationship between such paths and plant roots as well as provide a new way of quantitatively analyzing the horizontal space distribution characteristics of soil preferential flow paths.

\section{Conclusions}

The results indicate that an increase in water volume penetration affects these flow paths. This was reflected in the increase in the quantities of preferential flow paths of large size class in the topmost soil, as well as in the overall connectivity of paths. As per investigation into the spatial distribution of the preferential flow paths of horizontal stained profiles in different soil layers under HPWV and LPWV treatments, the paths in horizontal profiles exhibited a distinct clumped distribution at the soil profile scale $(x=25 \mathrm{~cm})$ in the study area. The differences in the spatial distributions of preferential flow paths were complicated with increased water volume. Such distributions tended to be uniform with spatial scale expansion. Thus, the spatial heterogeneity of forest soil structure is mainly related to distribution position and the configuration of the plants. Moreover, effective soil water conduction capability is sustained in the forest soil of the entire study area.

The spatial association of the preferential flow paths with plant roots showed a significant positive correlation in the different soil layers, especially under HPWV treatment, indicating that the formation of such paths in the topmost soil is primarily related to the growth of superficial plant roots. It displayed an insignificant correlation in the $50-\mathrm{cm}$ soil layer because the bed rock is severely weathered and fractured, and plant roots did not extend to this area.

These findings suggest that point pattern analysis is suitable for quantitatively analyzing the characteristics of the horizontal spatial distribution of preferential flow paths. This method can also be used to determine the relationship between such paths and plant roots.

\section{Acknowledgements}

We gratefully acknowledge the editor and reviewers. This research was jointly funded by the Fundamental Research Funds for the Central Universities (No. TD2011-2), the National Natural Science Foundation of China (Contract No. 30900866 and No. 41271301), Beijing Higher Education Young Elite Teacher Project (YETP0750) and the Special Fund of Forestry Industrial Research for Public Welfare of China (201204212).

\section{References}

Besag, J. and P.J. Diggle. 1977. Simple Monte Carlo tests for spatial pattern. Appl. Stat. 26: 327-333.

Bouma, J. 1981. Soil morphology and preferential flow along macropores. Agri. Water Manage. 3: 235-250.

Chao, W.C., S.H. Wu, S.W. Fan, H.Y. Lin, C.F. Hsieh and K.J. Chao. 2008. Distribution patterns of tree species in a lowland rainforest at Nanjen Lake. TAIWANIA. 53 (2): 124-133.

Cheng, J.H, J.D. Wu, Y.Z. Chen and H.J. Zhang. 2014. Characteristics of preferential flow paths and their effects on soil properties. For. Chron. 90 (2): 192-196. 
Cheng, J.H., H.J. Zhang, W. Wang, Y.Y. Zhang and Y.Z. Chen. 2011. Changes in preferential flow path distribution and it's affecting factors in Southwest China. Soil Sci. 176 (12): 22-27.

Cheng, J.H., H.J. Zhang, Y.H. Shi, F. He, S.L. Qi and Y.H. Sun. 2006. Affecting factors of preferential flow in forest of Three Gorges area,Yangtze River. J. Soil Water Conserv. 20 (5): 28-33.

Diggle, P.J. 1983. Statistical analysis of spatial point patterns. London: Academic Press.

Droogers, P., A. Stein, J. Bouma and G. De Boer. 1998. Parameters for describing soil macroporosity derived from staining patterns. Geoderma. 83: 293-308.

Gish, T.J., C.S. Helling and M. Mojasevic. 1991. Preferential movement of atrazine and cyanazine under field conditions. T. Am. Soc. Agri. Engin. 34: 1699-1705.

Gish, T.J., D. Gimenez and W.J. Rawls. 1998. Impact of roots on ground water quantities. Plant Soil 200: 47-54.

Goreaud, F. and R. Pélissier. 1999. On explicit formulas of edge effect correction for Ripley's K-function. J. Veg. Sci. 10: 433-438.

Haws, N.W., B.S. Das and P.S.C. Rao. 2004. Dual-domain solute transfer and transport processes: evaluation in batch and transport experiments. J. Contam. Hydrol. 75: 257-280.

Helling, C.S and T.J. Gish. 1991. Physical and chemical processes affecting preferential flow. Gish T.J., Shirmohammadi A. (eds.) Preferential Flow. Am. Soc. Agri. Engin. 77-86 (EI)
Lu, WL. 2009. Studies on forest plant community structure and biodiversity in Chongqing Simian mountain. Beijing Forestry University: 52-54.

Mitchell, A.R., T.R. Ellsworth and B.D. Meek. 1995. Effect of root systems on preferential flow in swelling soil. Commun. Soil Sci. Plan. 26: (15) 2655-2666.

Noguchi, S., Y. Tsuboyama and R.C. Sidle. 1999. Morphological characteristics of preferentials and the distribution of preferential flow pathways in a forested slope segment. Soil. Sci. Soc. Am. J. 63: 1413-1423.

Ripley, B.D. 1981. Spatial Statistics. 252 pp. Wiley, New York, USA. Vincent, L. and P. Soille. 1991. Watersheds in digital spaces: an efficient algorithm based on immersion simulations. IEEE. T. Pattern Anal. 13(6): 583-598.

Weiler, M. and H. Flïhler. 2004. Inferring flow types from dye patterns in macroporous soils. Geoderma. 120: 137-153.

Wiegand, T. and K.A. Moloney. 2004. Rings, circles, and null models for point pattern analysis in ecology. Oikos. 104: 209-229.

Zhang, C.Y. and X.H. Zhao. 2008. Spatial points pattern analysis using random square-quadrats method. Acta Ecologica Sinica. 7: 3108-3115. 